\title{
Syk Family Tyrosine Kinase
}

National Cancer Institute

\section{Source}

National Cancer Institute. syk Family Tyrosine Kinase. NCI Thesaurus. Code C19284.

The syk family tyrosine kinases are non-receptor protein tyrosine kinases structurally similar to the syk protein (spleen tyrosine kinase) containing two SH2 (SRC homology 2) domains. They play essential roles in a variety of immune and non-immune cells. 